\title{
Hepatitis C virus's next top models?
}

\author{
David Paul and Ralf Bartenschlager
}

\begin{abstract}
Advances in culturing hepatitis $C$ virus have given hope for a universal cell culture system amenable to primary isolate replication. However, low replication efficiency needs to be overcome. The development of fully susceptible yet immunocompetent in vivo models would aid research towards a prophylactic vaccine.
\end{abstract}

C hronic infection by hepatitis $\mathrm{C}$ virus $(\mathrm{HCV})$ is a major cause of liverassociated morbidity and mortality worldwide. Despite the tremendous advances made in the development of direct-acting antiviral drugs (DAAs) that target viral proteins, a rapid decline of the global disease burden is hampered by remarkably high treatment costs and a high number of undiagnosed infections ${ }^{1}$. Moreover, a fraction of patients develop resistance to therapy and a prophylactic vaccine is likely to be required for global $\mathrm{HCV}$ eradication. To address these aspects, novel cell culture systems supporting the replication of primary virus isolates in patient sera and fully permissive immunocompetent in vivo models are required. In vitro, a major challenge has been the inability of almost all patient isolates to replicate in (hepatoma) cell culture systems. This was overcome by the establishment of drug-selectable HCV mini-genomes termed replicons (Fig. 1a), which upon transfection autonomously replicate in cultured cells (reviewed in ref. 2). However, the selection process forces the accumulation of cell-cultureadaptive replication-enhancing mutations, which impair productive infection in vivo, probably due to a defect in progeny virus production $^{2}$. The only exception is the genotype 2 a isolate JFH1, which replicates to very high levels in cell culture without requiring such adaptive mutations ${ }^{3}$. This has fostered the use of the somewhat atypical JFH1 isolate, but prevented the study of clinical isolates in cultured cells, as the development of in vitro systems to allow efficient propagation of patient-derived $\mathrm{HCV}$ has remained a challenge. Attempts to render cells more permissive for $\mathrm{HCV}$ replication have generated highly selected cell clones ${ }^{4}$, such as Huh7.5, derived from the human hepatoma cell line Huh7. This clone allows efficient propagation of the
JFH1 strain, but fails to replicate patientderived virus or molecular HCV clones with proven in vivo infectivity.

The inability of HCV isolates to replicate in cultured hepatoma cells can be due to the absence of essential dependency factor(s) and/or the presence of one or several restriction factors. Comprehensive gain- and loss-of-function high-throughput screens in Huh7.5 cells allowed the recent identification of SEC14L2 as a factor that enables the replication of wild-type (WT) HCV replicon RNA (Fig. 1b) ${ }^{5}$. SEC14L2, a soluble lipid and vitamin E (VitE)-binding/ transport protein, is expressed in primary hepatocytes - which support replication of clinical HCV isolates - but not in various hepatoma cell lines, providing a rationale for the inability of WT HCV to replicate in the latter. Ectopic expression of SEC14L2 renders several hepatoma cell lines permissive for replication of subgenomic WT HCV RNAs derived from most genotypes, but has little effect on the replication of adapted RNAs or a JFH1 replicon. Although SEC14L2 renders Huh7.5 cells permissive for WT strains, transient replication assays revealed rather low replication efficiency. However, when transfected cells were subjected to selection, stable HCV-genome-containing cell clones that harboured high amounts of viral RNA were obtained. This suggests that either initial replication occurs only in a low number of cells that are enriched during the selection process, or that events other than the acquisition of adaptive mutations are required for efficient amplification of HCV RNA. In any case, full-length WT $\mathrm{HCV}$ strains produce infectious progeny virus in SEC14L2-expressing cells, but the low amounts of virus particles require detection by using a drug-resistance selection approach. Presumably for that reason, more direct measurements of virus production such as infectivity assays or the characterization of progeny virions were not reported. Importantly, SEC14L2expressing cells can be infected with $\mathrm{HCV}$ obtained from patient sera containing high viral titers, thus opening new avenues to study clinical HCV isolates. However, given the limited efficiency of infection, whether SEC14L2-expressing Huh7.5 cells infected with patient sera support progeny virus production remains unclear, as does whether this system can be used for clinical applications, such as phenotypic resistance assays.

\section{Novel cell culture systems supporting the replication of primary virus isolates in patient sera and fully permissive immunocompetent in vivo models are required.}

SEC14L2 does not seem to interact with viral replicase proteins ${ }^{5}$, although the twohybrid approach used to determine this is limited by the hydrophobic properties of the membrane-associated HCV replicase proteins. Nevertheless, SEC14L2 is not recruited to viral-protein-containing membrane fractions isolated from $\mathrm{HCV}$ replicon-containing cells $s^{5}$ and thus seems to play an indirect role in the enhancement of HCV replication. While several SEC14L2related functions do not seem to be required for the enhancement of HCV replication, its VitE binding ability appears to play a major role. Membrane-associated HCV replicase proteins are negatively regulated by lipid peroxidation ${ }^{6}$, which in turn is prevented by VitE. In support of SEC14L2 having a role in this process, its expression does not facilitate the replication of lipidperoxidation-insensitive HCV strains (for example, JFH1) and other viruses, but 
enhances that of the lipid-peroxidationsensitive H77S.3 strain. Although expression of SEC14L2 only moderately increases VitE accumulation, its presence further stimulates the SEC14L2-mediated positive effect on long-term replication.

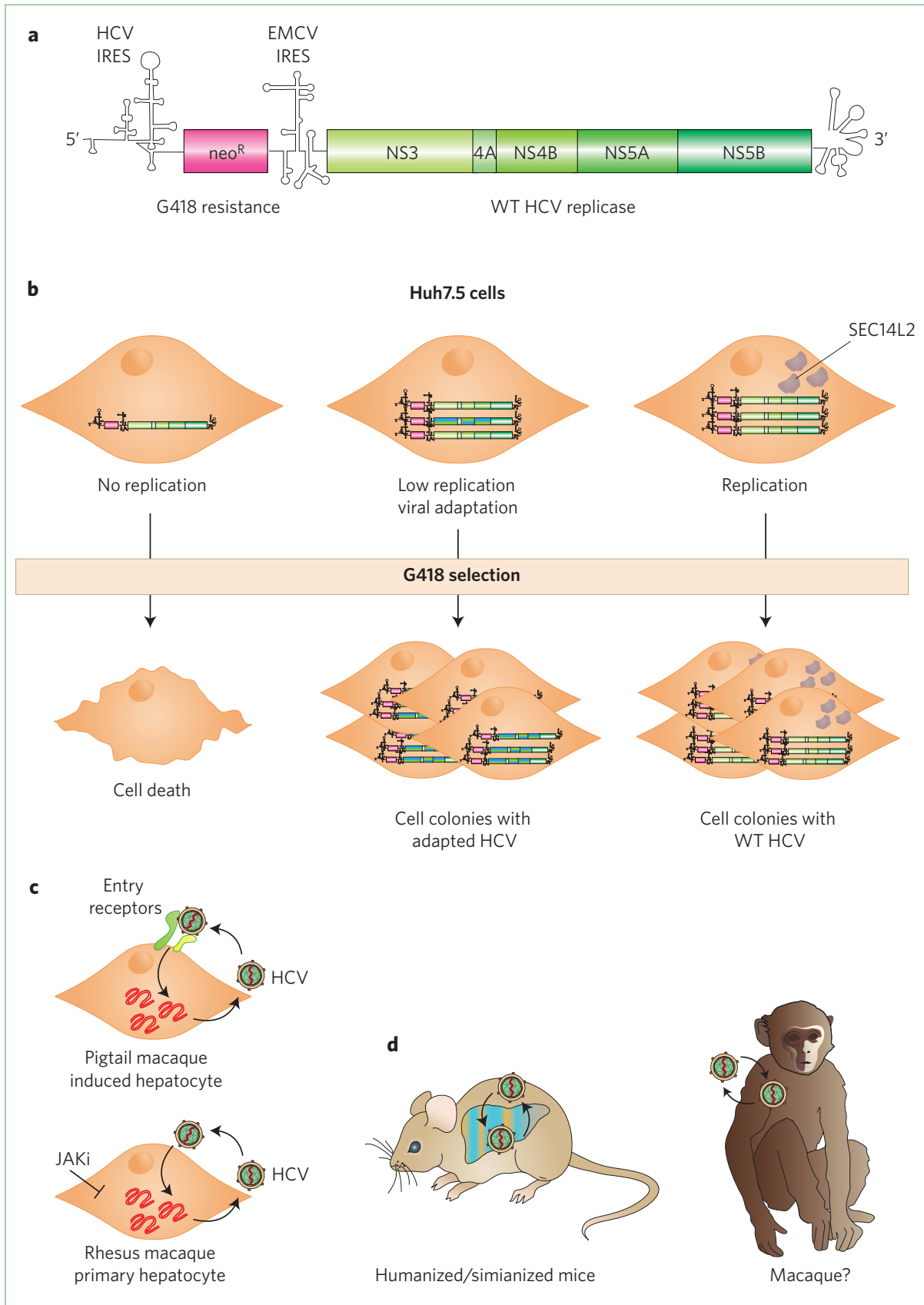

Figure 1 | Model systems for the study of HCV in vitro and in vivo. $\mathbf{a}$, Selectable subgenomic HCV replicon. The translation of the neomycin phosphotransferase $\left(\right.$ neo $\left.^{\mathrm{R}}\right)$ is under control of the $\mathrm{HCV}$ internal ribosome entry site (IRES), whereas the second cistron, comprising the viral replicase, is translated via the IRES of the encephalomyocarditis virus (EMCV). b. The HCV replicon system. Upon transfection of the selectable HCV replicon RNA, cells containing high amounts of actively replicating HCV RNA - enabled by the accumulation of replication-enhancing mutations - are selected through their antibiotic-resistant phenotype. Overexpression of SEC14L2 allows long-term replication of WT HCV RNA in cultured hepatoma cells. c, Pigtail-macaque-derived hepatocyte (like) cells overexpressing viral entry receptors, and primary rhesus-macaque hepatocytes can be infected with HCV. Viral replication in the latter is further boosted by inhibiting the antiviral immune response. $\mathbf{d}$, Immune-deficient mice with simian or human liver xenografts can be used to study persistent HCV infection invivo. However, the challenge to develop a fully permissive and immunocompetent macaque-based animal model remains.

Intriguingly, VitE on its own does not stimulate WT HCV long-term replication, implying that SEC14L2 has additional VitE-independent roles. Overall, SEC14L2 seems to facilitate $\mathrm{HCV}$ replication indirectly by rendering the cellular environment more conducive for $\mathrm{HCV}$ replication, for example by counteracting virus-induced, replication-limiting oxidative stress. Nevertheless, further biochemical and complementary imagingbased approaches are needed to better define the role of SEC14L2 in HCV replication and in host cell homeostasis in general. Additionally, the role of SEC14L2 lipid binding and possible lipid transfer properties should be analysed in the context of HCV replication. In all, although the identification of SEC14L2 lays the foundations for a long-awaited, universally suitable HCV cell culture system, the relatively low HCV replication efficiency, as determined by transient assays, suggests that additional factors are critically required to enhance HCV replication.

$\mathrm{HCV}$ has a narrow host range, infecting only humans and chimpanzees. This species specificity represents a major roadblock for pathogenesis and immune response studies, as well as for vaccine development. Notably, the murine SEC14L2 homologue also enables HCV replication, excluding this protein as a species-specific dependency factor ${ }^{5}$. Intensive efforts have been undertaken to render (immunocompetent) laboratory mice permissive for $\mathrm{HCV}$, but this has so far met only limited success ${ }^{7}$. One alternative is to search for animal models that are more closely related to humans. Following this rationale, two groups have recently demonstrated that cells derived from pigtail and rhesus macaques (Macaca nemestrina and mulatta, respectively) are permissive for $\mathrm{HCV}$ infection, in contrast to the previously reported ${ }^{8}$ inability of HCV to infect rhesus macaques (Fig. 1c). Engineered hepatocyte-like cells from pigtail macaques overexpressing HCV entry factors were able to sustain the entire viral replication cycle of JFH1-derived HCV in cell culture ${ }^{9}$, indicating an apparent block in the early stages of the viral life cycle in this system. In the second study, rhesus-macaque-derived primary hepatocytes were productively infected by a HCV genotype 2a JFH1-based reporter virus ${ }^{10}$. Simian orthologues of $\mathrm{HCV}$ entry factors were shown to support viral uptake, and HCV propagation could be stimulated by blunting host immune signalling. Notably, HCV of genotypes 1a and 2 a could infect immunodeficient mice harbouring macaque hepatocyte xenografts (Fig. 1d). This simianized mouse model allowed in vivo mimicking of a persistent 
$\mathrm{HCV}$ infection, albeit with lower efficiency as compared with human-liver chimaeric mice. Collectively, these data suggest that macaques might be a promising model system to study HCV infection in vivo.

These are encouraging developments in our ability to study primary HCV isolates in vitro and in vivo. However, if these new model systems are to support studies to develop prophylactic vaccines for $\mathrm{HCV}$, major hurdles - such as productive HCV infection in the presence of a functional host immune defence - remain to be overcome.

David Paul and Ralf Bartenschlager are in the Department of Infectious Diseases, Molecular Virology, University of Heidelberg, 69120 Heidelberg, Germany. Ralf Bartenschlager is also in the Division of Virus-Associated Carcinogenesis, German Cancer Research Center (DKFZ), 69120 Heidelberg, Germany.

e-mail: ralf_bartenschlager@med.uni-heidelberg.de

\section{References}

1. Hill, A. \& Cooke, G. Science 345, 141-142 (2014).

2. Lohmann, V. \& Bartenschlager, R. J. Med. Chem. 57, 1627-1642 (2014)

3. Wakita, T. et al. Nature Med. 11, 791-796 (2005).

4. Blight, K. J., McKeating, J. A. \& Rice, C. M. J. Virol. 76, 13001-13014 (2002).

5. Saeed, M. et al. Nature 524, 471-475 (2015).

6. Yamane, D. et al. Nature Med. 20, $927-935$ (2014).

7. Vercauteren, K., de Jong, Y. P. \& Meuleman, P. Curr. Opin. Virol. 13, 67-74 (2015).

8. Bukh, J. et al. J. Viral Hepat. 8, 228-231 (2001).

9. Sourisseau, M. et al. Gastroenterology 145, 966-969 (2013).

10. Scull, M. A. et al. Hepatology 62, 57-67 (2015). 